\title{
Methylprednisolone Pulse Therapy Can Be Life-Saving in Neonatal Lupus Erythematous: A Case Report
}

\author{
Maliheh Kadivar, ${ }^{1,2}$ Razieh Sangsari, ${ }^{1,2,}$ Leila Shahbaznejad, ${ }^{3}$ and Vahid Ziaee ${ }^{1,2,3}$ \\ ${ }^{1}$ Department of Pediatrics, Tehran University of Medical Sciences, Tehran, Iran \\ ${ }^{2}$ Children's Medical Center, Pediatrics Center of Excellence, Tehran, Iran \\ ${ }^{3}$ Pediatric Rheumatology Research Group, Rheumatology Research Center, Tehran University of Medical Sciences, Tehran, Iran \\ "Corresponding author: Dr. Razieh Sangsari, Children’s Medical Center, 62 Gharib Street, 1419733151 Tehran, Iran. Tel: +98-2166920983, E-mail: raz3532@yahoo.com
}

Received 2016 September 17; Revised 2016 December 03; Accepted 2017 January 13.

\begin{abstract}
Introduction: Neonatal lupus erythematous (NLE) is a neonatal autoimmune disease, due to passage of maternal autoantibodies against Ro/SS-A, La/SS-B, and U1-ribonucleoprotein, with multi organ involvement. Although the clinical manifestations of NLE include cutaneous, cardiac, hematologic, and hepatobiliary system involvement, the most common clinical manifestations are skin rash and heart involvement. Congenital heart block in the absence of structural cardiac abnormalities is the most commonly observed manifestation. The most clinical manifestations of NLE are self-limited and resolve without serious treatment, except heart block that may need a pacemaker. In rare instances, more severe complications may occur, and necessitate more aggressive immune suppressive therapy.

Case Presentation: Here we present a neonate girl who developed cutaneous, cardiac, liver, brain, splenic, kidney, and hematologic abnormalities. After 2 months of aggressive immune suppressive therapy she could be discharged. The treatment was tapered stepwise and discontinued at the age 1 year. At the age of 4 years she was in a favorable health condition.
\end{abstract}

Keywords: Neonatal Systemic Lupus Erythematous, Infant, Autoimmune Disease

\section{Introduction}

Neonatal lupus erythematous (NLE) is an autoimmune disease in neonatal period (1). It is caused by transplacental passage of maternal autoantibodies against Ro/SS-A, La/SS$\mathrm{B}$, and U1 ribonucleoprotein (2). Although skin rash and congenital heart disease are the most common manifestations of the disease, it has systemic involvements (3). Cutaneous rashes often develop within a few weeks after delivery, especially when sun exposed to sunlight, but may be present at birth (1). Congenital heart block without structural disease is most commonly presented as cardiac manifestation (4). Congenital heart block may be present in the fetal or neonatal period (1). The diagnosis is usually established based on the clinical features and NLEassociated antibodies in the serum of the affected infant or the mother (5). The condition is usually benign and self-limited and resolves spontaneously along with disappearance of maternal antibodies in the neonatal circulation, however, it sometimes may be associated with permanent sequels such as in heart disease (2). Infants with severe hepatic and hematological involvement may require treatment with systemic corticosteroids, intravenous immunoglobulin, and/or immunosuppressive agents (5).

\section{Case Presentation}

Here we report a case of NLE with multi organ involvement and more severe complications that was treated by aggressive immune suppressive therapy.

A 22-day-old female newborn infant was referred to the neonatal intensive care unit of our tertiary referral center in Tehran due to prolonged hyperbilirubinemia and skin lesions presenting from the first day of life. She was born by a cesarean section at the gestational age of 38 weeks due to meconium staining of amniotic fluid during labor. Birth weight, head circumference, and height was, 2050 grams, $34 \mathrm{~cm}$, and $48 \mathrm{~cm}$, respectively. The apgar score of 8 and 10 at 1 and 5 minutes after birth. She was the first child of a 25 -year-old mother. The mother had not experienced any complication during her uneventful pregnancy despite mild thrombocytopenia (platelet count was nearly $90000 / \mathrm{mm}^{3}$ ), which was thought to be pregnancy induced thrombocytopenia and remained untreated without further investigation.

On the physical examination, the infant was greenish yellow in color and had annular erythematous rashes on her face, trunk, and all limbs. The rash and yellow color appeared in the first days of life. She also had moderate hepatosplenomegaly and a heart murmur.

The results of the hematologic analysis revealed pan- 
cytopenia with reticolucytosis and positive direct Coombs test. Hepatobiliary system screening showed direct hyperbilirubinemia, elevated liver transaminases, and alkaline phosphatase (Table 1). Serologic investigation for cytomegalovirus (CMV), toxoplasmosis, syphilis, rubella, and human immunodeficiency virus (HIV) was negative. A sepsis work up including blood and urine culture was also negative.

Table 1. Laboratory Characteristics of Patient and Her Mother at the Time of Admission and During Follow Up

\begin{tabular}{|c|c|c|c|c|}
\hline \multirow[t]{2}{*}{ Variables } & \multirow[t]{2}{*}{ Mother } & \multicolumn{3}{|c|}{ Infant } \\
\hline & & admission & $\begin{array}{l}3.5 \text { month } \\
\text { old }\end{array}$ & 6 month old \\
\hline WBC, $\mathbf{1 0}^{3} / \mu \mathbf{L}$ & 7200 & 4520 & 6000 & 7930 \\
\hline $\mathbf{P M N}, \mathbf{1 0}^{3} / \mu \mathbf{L}$ & & 1800 & 3070 & 2470 \\
\hline $\mathbf{L Y M}, \mathbf{1 0}^{3} / \mu \mathbf{L}$ & & 2300 & 2060 & 4360 \\
\hline Mon, $\mathbf{1 0}^{3} / \mu \mathbf{L}$ & & 370 & 860 & 920 \\
\hline Eos, $\mathbf{1 0}^{3} / \mu \mathbf{L}$ & & 30 & 10 & 30 \\
\hline Hgb, g/dL & 11 & 8 & 9.9 & 12.6 \\
\hline Hct, \% & & 25 & 29 & 37 \\
\hline Plt, $\mathbf{1 0}^{3} / \mu \mathbf{L}$ & 50000 & 35000 & 267000 & 253000 \\
\hline Bill T, mg/dL & & 14.7 & 18.8 & 0.6 \\
\hline Bill D & & 8 & 9.5 & 0.2 \\
\hline AST, IU/L & & 967 & 880 & 41 \\
\hline ALT, IU/L & & 284 & 580 & 67 \\
\hline ALP, IU/L & & 1151 & 888 & 667 \\
\hline $\begin{array}{l}\text { Anti RO, } \\
\mathbf{u} / \mathbf{m l}\end{array}$ & $>100$ & $>100$ & & 5 \\
\hline Retic,\% & & 7.8 & & \\
\hline
\end{tabular}

Abbreviations: ALP, Alkaline Phosphatase; ALT, Alanine Transaminase; AST, Aspartate Transaminase; Bill D, Direct Bilirubin; Bill T, Total Bilirubin; Eos, Eosinophil; Hct, Hematocrit; Hgb, Hemoglobin; LYM, Lymphocyte, Mon, Monocyte; Plt, Platelet; PMN, Polymorphonuclear Leukocytes; Retic, Reticulocyte; WBC, White Blood Cell.

Echocardiography revealed moderate mitral regurgitation, which was due to perforation in posterior leaflet of mitral valve, accompanied with mild tricuspid regurgitation and patent ductus arteriosus (PDA) with left to right shunt. She had neither signs of arrhythmia nor heart block in further evaluation.

Later, an episode of seizure accompanied by repeated apneas led to tracheal intubation and mechanical ventilation. A brain ultrasonography revealed hyperechogenicity in bilateral thalamic and putamen areas. These findings might be due to either vasculitis or asphyxia. The kidney ultrasonography also showed bilateral hyperechogenicity. Retinal examination was normal.

During admission, she received phenobarbital for seizures and antibiotics for sepsis.

Laboratory investigation in the mother revealed positive ANA, RO/SSA, LA/SSB, Anti double stranded DNA, antiphospholipid and indirect Coombs test. In addition, the mother low titers of $\mathrm{C} 3$ and $\mathrm{C} 4$ complement. The mother did not have any clinical presentation of systemic lupus erythematous (SLE) in her past history, though she had been referred to an adult rheumatologist for further investigation for SLE and follow up.

The histopathological analysis of skin biopsy in the neonate revealed basal cell vacuolization of epidermis and dermis with mild perivascular infiltration of mononuclear cells. Focus of follicular plugging and apoptotic cells were also noted (Figure 1).

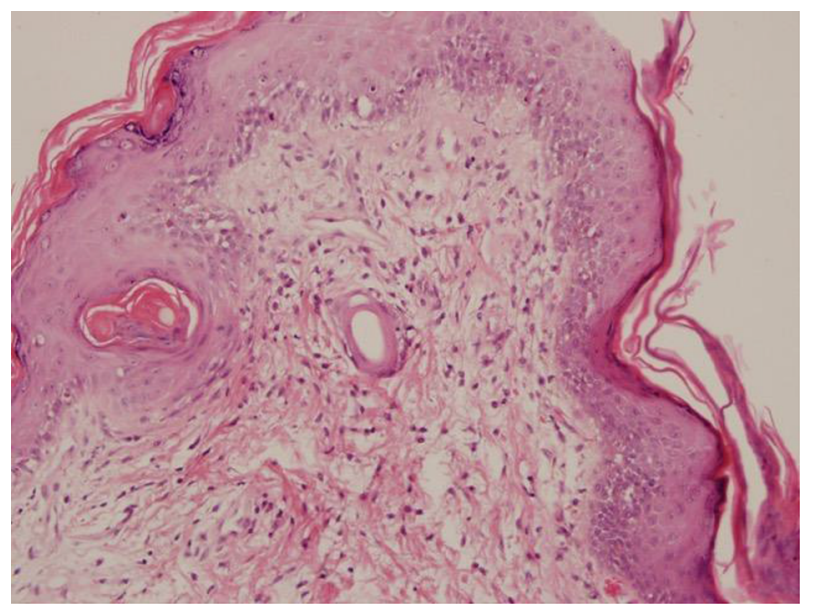

Figure 1. Sections Show Skin Tissue with Basal Layer Vacuolization and Focal Apoptosis. Mild Perivascular Lymphocytic Infiltration and Focal Follicular Plugging Are Also Noted. (Hematoxillin and Eosin, 400X)

Establishing the diagnosis neonatal lupus, treatment started with ursodeoxycholic acid (Ursobil), zinc supplement, folic acid, fat-soluble vitamins, topical corticosteroid, sunscreen ointment, and systemic hydrocortisone. Moreover, the patient received intravenous immunoglobulin (IVIG) due to her poor general condition, thrombocytopenia, and cardiac involvement, without satisfactory improvement.

Respiratory distress and seizures continued to exist. The disease worsened. Infectious disease was ruled out. We began methyl prednisolone pulse therapy with 30 $\mathrm{mg} / \mathrm{kg} /$ day, 3 consecutive days, followed by a high dose of corticosteroid ( $1 \mathrm{mg} / \mathrm{kg} /$ day prednisolone). On this treatment, the general condition of the patient improved and she could be weaned from mechanical ventilation during 2 days. Figure 2 shows her general appearance after extubation. 


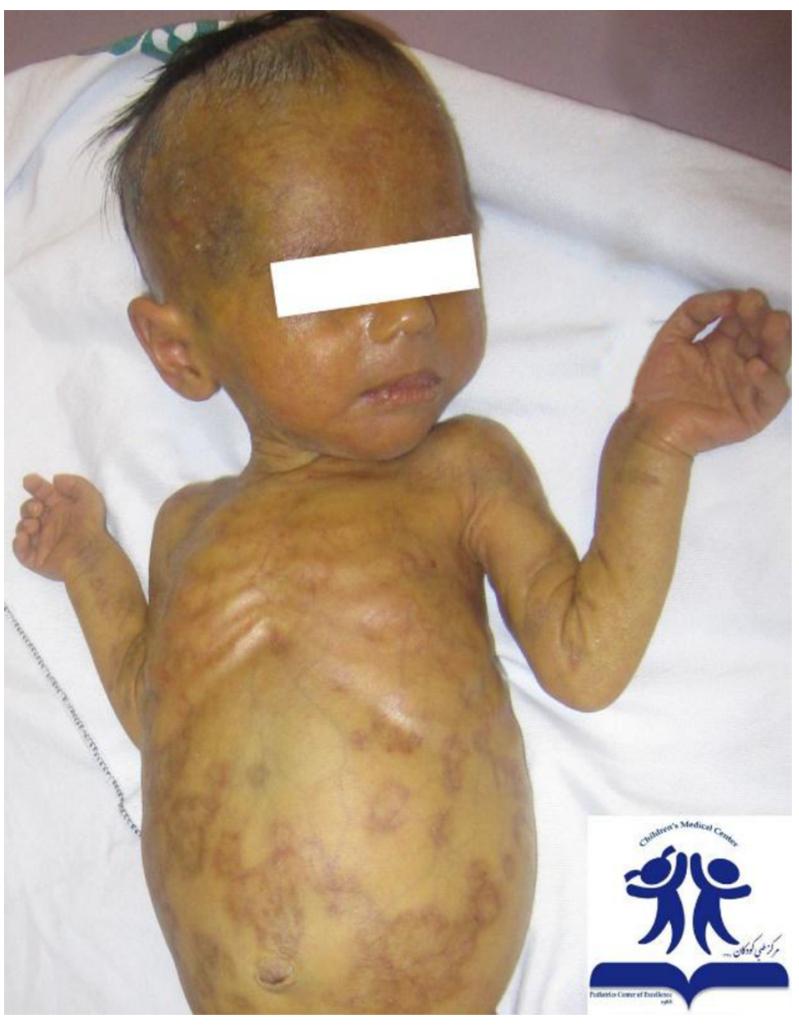

Figure 2. Annular Skin Rashes Over the Face, Trunk and Extremities. Note to the Icteric Color of the Skin (Photo on Admission After Improvement of General Condition and Extubation)

Echocardiography under immune suppressive therapy revealed mild mitral regurgitation with good cardiac contractility. The hematologic values gradually became normal with normalization of aminotransferases and bilirubin levels. The seizure was controlled so that phenobarbital could be tapered during 3 months. The skin rashes healed with hypopigmentation and residual atrophic areas. The patient was discharged after 2 months with corticosteroid prescription. After the 6 months follow up, the patient showed complete resolution of the findings, good general condition, and normal development. Thus, all treatment was tapered stepwise and discontinued at 1 year old. Figure 3A shows the 1-year-old patient with mild hypopigmentation and telangiectasia. After 4 years, these findings were completely resolved and the development was normal (Figure 3B).

\section{Discussion}

Neonatal lupus is a rare acquired autoimmune disease caused by the transplacental transfer of maternal auto antibodies, characterized by transient or permanent manifestations (6). This autoimmune disorder occurs in neonates of mothers with SLE but it may be seen in other connective tissue disorders such as Sjogren syndrome and even rheumatoid arthritis (7). The most common clinical manifestations of NLE are heart block (50\%), dermatological manifestations (34\%), and a coincidence of the 2 symptoms in $10 \%$ of cases (6). Other manifestations include hematological, neurological, kidney or splenic abnormalities (1). Although dermatological manifestations commonly occur in exposed areas after sun exposure, it may also be seen immediately after birth without any sun exposure (2). This manifestation is transient and rarely leaves atrophic scars (2). Cardiac disease with third-degree heart block is the most common cardiac manifestation, however, the other less common manifestations are myocarditis, cardiomyopathy often with endocardial fibroelastosis, and valvular lesions causing stenosis, regurgitation, and dysplasia due to inflammation and fibrosis (1). Hepatobiliary manifestation is seen as hypertransaminasemia, however, hepatosplenomegaly and cholestasis are less common (8). Although CNS involvement is generally asymptomatic (4), focal clonic seizures with ischemic brain lesions have been reported (9). Hydrocephaly, calcification of the basal ganglia, and vasculopathy are also reported (10). Hematologic findings including hemolytic anemia, thrombocytopenia, and leukopenia are other transient manifestations $(2,11)$.

Our patient presented cutaneous, cardiac, liver, brain, kidney, splenic, and hematologic abnormalities. Cardiac manifestation consisted of mitral regurgitation caused by a perforation in posterior leaflet of the mitral valve. This was due to inflammation that could be reduced with immune suppressive therapy (1). The cutaneous lesions occurred on the first day of life without exposure to sunlight. Hepatobiliary manifestations included elevation of transaminases, cholestatic jaundice and hepatomegaly. Brain involvement was presented as seizures followed by apnea attacks, which led to endotracheal intubation. Thalamus and putamen area were involved due to vasculitis. Enlargement of the spleen was a sign of its involvement. Kidney problem was shown as bilateral hyperechogenicity in sonography. These hyperechogenic lesions could be signs of fibrotic scars resulting from some type of inflammation, which responded to immune suppressive therapy (1). Other manifestations of hematologic, brain, liver, and splenic involvement responded to immune suppressive therapy as well.

Neonatal Lerythematosus should be considered in neonates with atypical organ manifestations especially with skin rash. Appropriate work-up includes EKG and serological evaluation.

Although the most common clinical manifestations in 

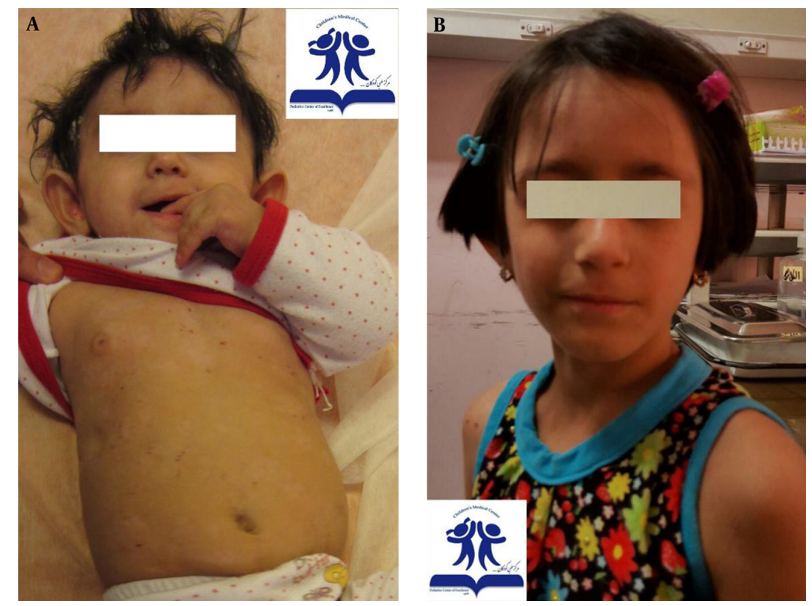

Figure 3. A, Mild Hypopigmentation with Telangectasia After 1 Year (Left); B, Complete Resolution of All Symptoms and Normal Development After 4 Years Follow Up (Right)

NLE are skin rash and heart block, multi system abnormalities may be seen. NLE signs may be self-limited manifestations, however, in some instances, more severe complications may occur, which need more aggressive immune suppressive therapy. Cardiac manifestations can improve with treatment.

\section{Acknowledgments}

We are grateful to Dr. Mehrzad Mehdizadeh (radiologist), Dr. Soheila Sotudeh (dermatologist), Dr. Maryam Monajemzadeh (pathologist), and Dr. Pari Zarrini (neonatologist) for technical help.

\section{References}

1. Morais S, Santos IC, Pereira DF, Mimoso G. Neonatal lupus with atypical cardiac and cutaneous manifestation. BMJ Case Rep. 2013;2013 doi: 10.1136/bcr-2013-009249. [PubMed: 23839605].

2. Hon KL, Leung AK. Neonatal lupus erythematosus. Autoimmune Dis. 2012;2012:301274. doi: 10.1155/2012/301274. [PubMed: 22973504].

3. Admani S, Krakowski AC. Neonatal Lupus Erythematosus Presenting as Atypical Targetoid-like Lesions Involving Genitals and Soles of Feet Following Brief Sun Exposure. J Clin Aesthet Dermatol. 2013;6(5):19-23. [PubMed: 23710267].
4. Buyon JP, Rupel A, Clancy RM. Neonatal lupus syndromes. Lupus. 2004;13(9):705-12. doi: 10.1191/0961203304lu20080a. [PubMed: 15485109].

5. Wisuthsarewong W, Soongswang J, Chantorn R. Neonatal lupus erythematosus: clinical character, investigation, and outcome. Pediatr Dermatol. 2011;28(2):115-21. doi: 10.1111/j.1525-1470.2011.01300.x. [PubMed: 21362029].

6. Trevisan F, Cunha PR, Pinto CA, Cattete FG. Cutaneous neonatal lupus with cutis marmorata telangiectatica congenita-like lesions. An Bras Dermatol. 2013;88(3):428-31. doi: 10.1590/abd1806-4841.20131832. [PubMed: 23793203].

7. Assari R, Ziaee V, Moradinejad MH, Mirmohammadsadeghi A. Neonatal Lupus erythematosus Following Rheumatoid Arthritis: Case Report and Literature Review. Iran J Pediatr. 2014;24(4):445-8. [PubMed: 25755869].

8. Singalavanija S, Limpongsanurak W, Aoongern S. Neonatal lupus erythematosus: a 20-year retrospective study. J Med Assoc Thai. 2014;97 Suppl 6:S74-82. [PubMed: 25391176].

9. Doring M, Rohrer KM, Tsiflikas I, Buchenau W, Wilke M, Handgretinger R, et al. A newborn with grouped facial skin lesions and subsequent seizures. BMC Pediatr. 2014;14:126. doi:10.1186/1471-2431-14-126. [PubMed: 24884686].

10. Silverman E, Jaeggi E. Non-cardiac manifestations of neonatal lupus erythematosus. Scand J Immunol. 2010;72(3):223-5. doi: 10.1111/j.1365 3083.2010.02443.x. [PubMed: 20696019].

11. Li L, Dong GF, Han FZ, Cui Y, Shi YZ, Zhang X. [Neonatal lupus erythematosus: a report of 7 cases and review of 87 cases of China]. Zhonghua Er Ke Za Zhi. 2011;49(2):146-50. [PubMed: 21426696]. 